\title{
E-CTP: An Energy-balanced Collection Tree Protocol for Power Constrained Wireless Sensor Networks
}

\author{
Jingjing Zhang ${ }^{1}$, Zhenqi Yang ${ }^{1,2}$, Baowei Wang ${ }^{1,2}$, \\ Huiyu $\operatorname{Sun}^{3}$ and Xingming $\operatorname{Sun}^{1,2}$ \\ ${ }^{1}$ School of Computer and Software, Nanjing University of Information Science \& \\ Technology, Nanjing 210044, China \\ ${ }^{2}$ Jiangsu Engineering Center of Network Monitoring, Nanjing 210044, China \\ ${ }^{3}$ Department of Mathematical Sciences, University of Bath, Bath BA2 7AY, \\ United Kingdom \\ jsycniga@gmail.com,to_yzq@163.com,wbw.first@163.com, \\ huiyu.sun@yahoo.co.uk,sunnudt@163.com,
}

\begin{abstract}
In Wireless Sensor Networks (WSNs), as the network consists of low-cost sensor nodes with finite battery power, power efficient strategies must be applied for data transmission in order to prolong the lifetime of the network. It is important to minimize the total energy consumption in each round so that the network lifetime is maximized. In this study, we propose a new energy efficient protocol E-CTP based on the Collection Tree Protocol (CTP). Taking into account the power available to the whole network and single node, the Expected Transmission Value (ETX) was updated according to the proposed protocol. The related experiments were carried on TelosB, the results were compared in terms of lifetime and efficiency. We conclude that the new protocol has a longer lifetime and higher efficiency.
\end{abstract}

Keywords: E-CTP; CTP; energy-balanced; WSN

\section{Introduction}

A wireless sensor network(WSN)[1-4] is composed of thousands of wireless sensors that are low-cost and multifunctional. As a fusion of sensor technology and embedded computing technology, a WSN can be controlled and queried by an external device (sink or based station) to detect, record, and transmit environmental information like temperature, humidity and illumination through multiple independent hops. The WSN is an ideal solution for collecting data in a variety of environments. In recent years, advances in the wireless technology have led to the development of WSN that can be used for many applications such as environmental monitoring, outdoor industrial and military surveillance. But the WSN nodes are also an example of energy constrained devices.

One of the most promising data collection protocol for WSN is the Collection Tree Protocol (CTP) [5-11]. CTP is a tree based collection protocol renowned for its reliability, robustness, efficiency, and hardware independence. Though the CTP contains the key principles of a collection protocol, it doesn't take into account the energy aspect of nodes in the network [12]. When the nodes are deployed in the network in different positions, the task of forwarding packets from those positions would be different from each other resulting in some nodes' energy consuming faster than others [13]. Especially in the existing meteorological sensors-some sensors, like the carbon dioxide sensor, temperature sensor and 
humidity sensor, are supplied by 2 AA batteries while barometric pressure sensor and pyranometer have an external power source such as solar cell. Sensors with external power supply should be selected to transmit information more frequently to prolong the lifetime of the entire network $[14,15]$.

In order to solve this problem, E-CTP is proposed in this paper, which takes the residual voltage into consideration. In Section 2, we will describe the related work in CTP; Details of E-CTP would be introduced in section 3; in Section 4, experiment based on TelosB [16]would be designed and related results are listed and discussed in terms of lifetime and voltage balance; Finally, several concluding remarks are listed in Section 5.

\section{Related Work}

E-CTP makes improvements upon the CTP. CTP is an address-free protocol in which an arbitrary node does not send information packet to a particular node. Instead, it chooses its root implicitly by selecting the next hop based on a rooting gradient, Which is called Expected Transmission Value(ETX) [17] indicating the link quality of the selected path to the root node. In CTP network, each node maintains EXT value for its neighbor nodes, and the neighbor with the minimum ETX value would be chosen as the next hop. There exist 3 parts in implementing the CTP protocol, which could be summarized as link estimator, routing engine and forwarding engine. The link estimator provides the interface for routing engine to compute the ETX value. Routing engine, in the process of transmitting to the root node, uses the information provided in the link estimator to choose the node with least cost as the parent node. Forwarding engine maintenances a send queue which contains local packets and received packets, and it selects an appropriate time to send the head packet of the queue to the parent node. In CTP, there are two methods for estimating link quality, i.e., the traditional LE and a more accurate realization, 4 BITLE [18]. Though 4bit link estimation has the same architecture with the traditional method, it has a more accurate behavior. Because it has extra information from physical layer, link layer and network layer. Figure 1 shows the relationship between 4 bit information and layers.

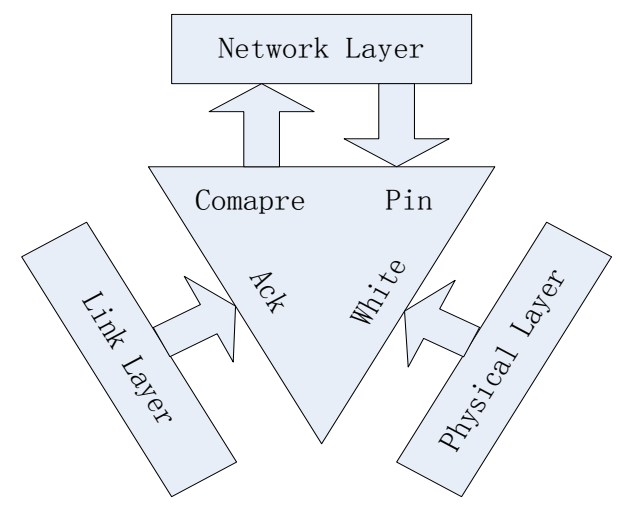

Figure 1. Model of 4bit Information

\subsection{Physical Layer}

The quality of the transmission channel could be approximated at physical layer .A received package with few bit errors would have better performance. As the measurement on physical layer can be completed rapidly with low-cost, the time wasted on inferior quality connection and on WSH margin could be avoided to a large extend. In the following work, we would use a "white" bit to express the channel quality. 


\subsection{Link Layer}

The acknowledgment and the confirmation of message transmission are achieved at link layer. Data loss is a severe problem resulted from the unstable quality and node failures in wireless sensor network .If data loss happens, it would be almost impossible for the link estimation to identify in time based on square detection, which separates the link layer estimation from data communication. To solve the inconvenience, a'ACK' bit was used to indicate the confirmation from the link layer in a single transmission.

\subsection{Network Layer}

The most valuable connection can be obtained at network layer. The net layer is proposed to overcome the drawback of the link estimation, such as its inability to handle node failure and suffering from routing loop.

As a result, the link estimator could describe a more accurate and efficient estimator by using the four bit link estimator interface.

\section{Design of E-CTP}

In this section, E-CTP would be discussed in details. Apart from 4bit information in traditional protocol, voltage information is also taken into consideration when estimating the link quality to prolong the lifetime of the WSN.

In the CTP, When the estimator receives a network layer routing packet which has the white bit set from a node that is not in the link estimation table and the compare bit is set, the estimator choose a random unpinned entry from the table and replaces it with the sender of the current packet. The estimator uses the ACK bit to refine the link estimates, combining broadcast and unicast ETX estimates into a hybrid value. We calculate separately the ETX value for every $K_{u}$ or $K_{b}$ packets for unicast and broadcast packets, respectively. If a out of

$K_{u}$ packets are acknowledged by the receivers, the unicast ETX estimate is $\frac{K_{u}}{a}$. If $a=0$, then the estimate is the last successfully delivered number. Compared with unicast packets, the calculation for the broadcast use a windowed exponentially weighted moving average over the calculated reception probabilities, and invert the consecutive samples of this average into ETX values. When there is heavy data traffic, the unicast estimates dominate. When the network is quiet, the broadcast estimates dominate.

E-CTP makes improvements in the field of link estimation for the wireless sensor based on CTP, which also consider the residual energy as an important factor to evaluate the quality of the link in addition to the original 4 Bit estimator.In E-CTP, when there is a large difference between the neighbor nod and the next nod, the algorithm will recalculate a new nod to balance the energy consume of the entire network.

Based on the 4Bit estimator the energy balance procedure could be divided into three parts, which would be explained in details as follows:

\subsection{Getting the Remaining Energy of each Node}

In this paper, our work is based on the TinyOS [19] , an Operating system written in NesC [20]. TinyOS is a kind of operating system including many components, e.g., main module, application module, process module and so on. A complete application consists of a schedule module, which acts as the essential component, and some other related components, which 
could be added up in when necessary in order to decrease the RAM. In this part, we use the interface of VoltageC to get the value of sensor's available power.

\subsection{Evaluate the Energy}

Considering characteristics of each node, there exists a voltage $V_{L}$. When the node's voltage is under $V_{L}$, the data collected would be meaningless though the node is still able to collect information,. Therefore we define the interval between $V_{\max }$ and $V_{L}$ as the normal operating voltage. Meanwhile a threshold, $V_{T}=\frac{V_{\max }+V_{L}}{2}$, is set as the alert voltage .

Also, we define a coefficient to maintain a better voltage balance.

$$
\delta=\left|\frac{U-\mu}{\sigma}\right|_{(1)}
$$

Where $\delta$ is the convergence coefficient, $u$ is the average value of all nodes, $U$ is the voltage of the particular sensor node, and $\sigma$ is the variance.

If the voltage value we got is less than $V_{T}$, we multiply the 4 bit estimator by the coefficient $\delta$ to get the ETX.

The E-CTP can be expressed as Figure 2:

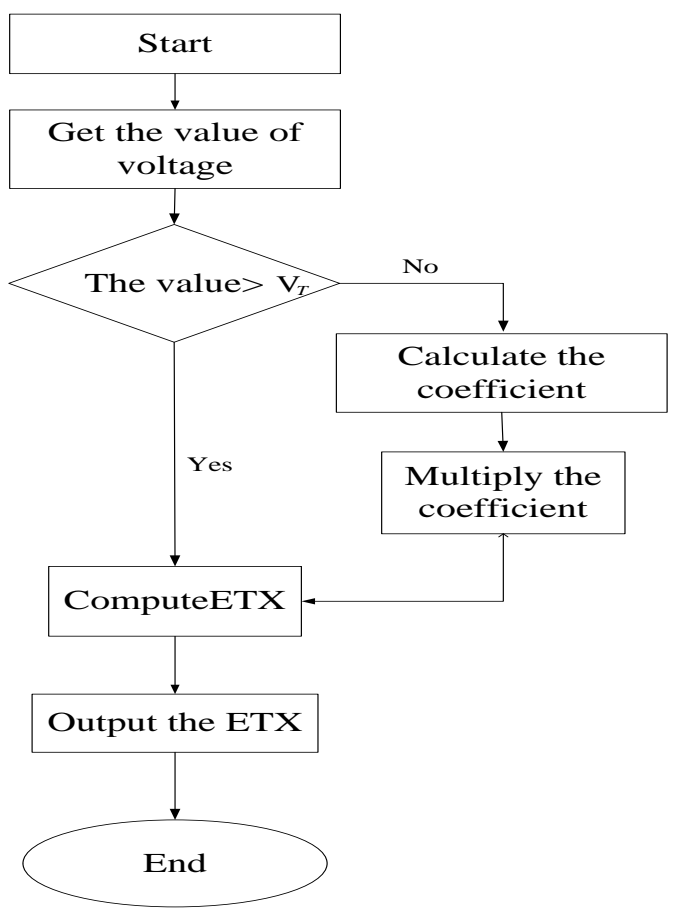

Figure 2. Flowchart of E-CTP 


\section{Experiments}

\subsection{Testbeds}

To evaluate the performance of E-CTP, an experiment was carried on a WSN which consist of TelosB sensor nodes while the sink node is connected to a computer, which is used to receive and calculate the data.

TelosB is an instrument widely used in low power consumption wireless sensor experiment. The structure could be seen in Figure 3, in which Data can be obtained through USB interface while TI MSPS430 10Kb Ram is used to control the whole operation and deal the Binary information from itself and other nodes. The sensor mode is used to collect the temperature, humidity and light information while the wireless module is responsible for communicating with other connection nodes to exchange data. The architecture can be expressed as follows:

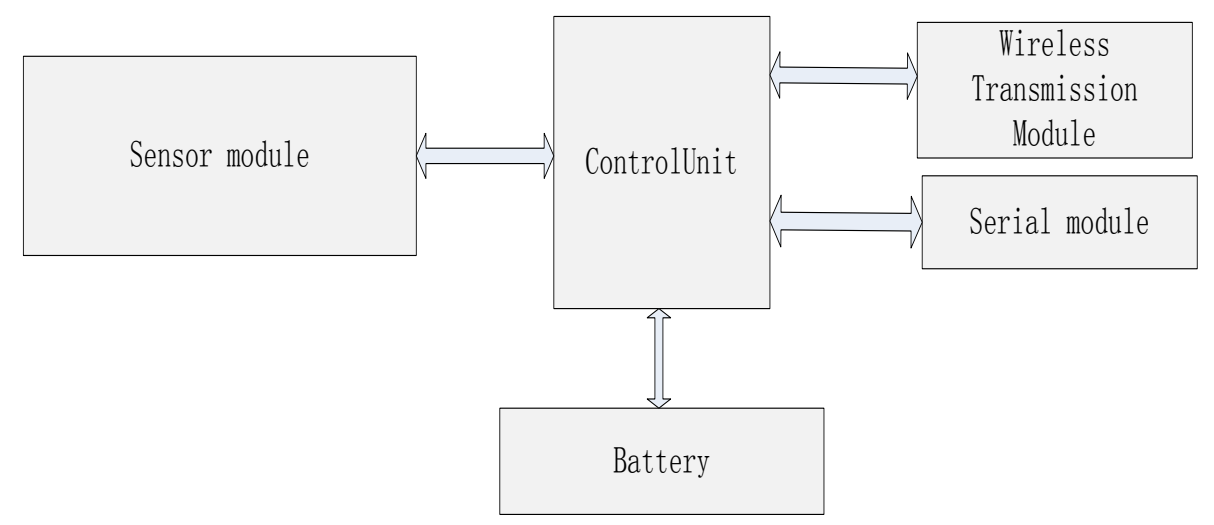

Figure 3. Structure of TelosB

At last, to satisfy the requirements of collection of relevant information, the deployment was completed as Figure 4 and Figure 5, in which in which the node numbered 0 performs as sink node, and the other nodes act as sensor nodes.

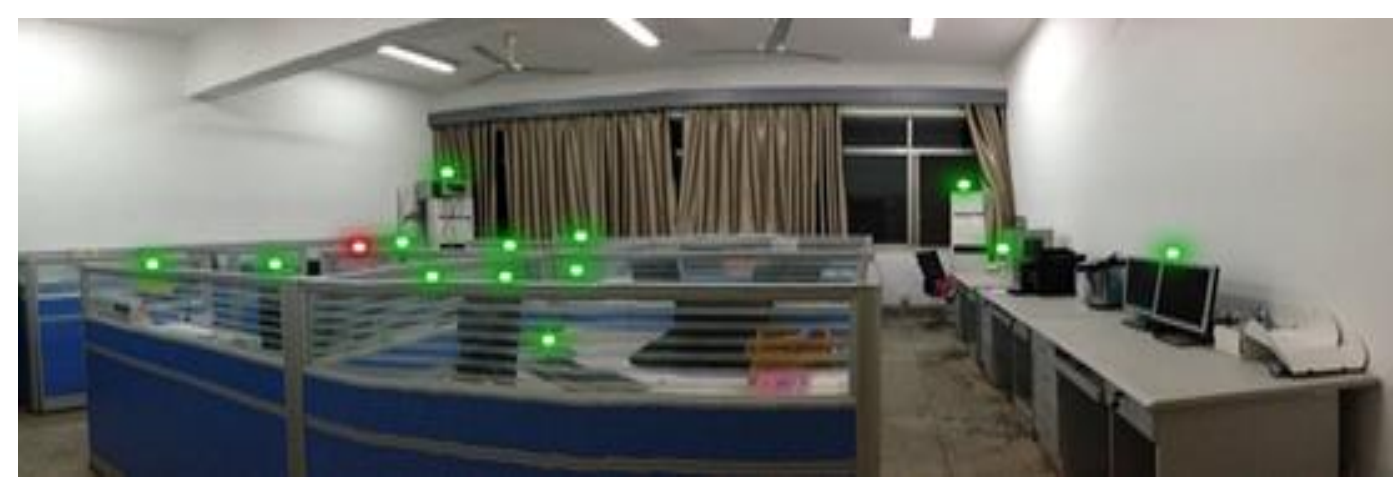

Figure 4. The Environment of Node's Deployment 


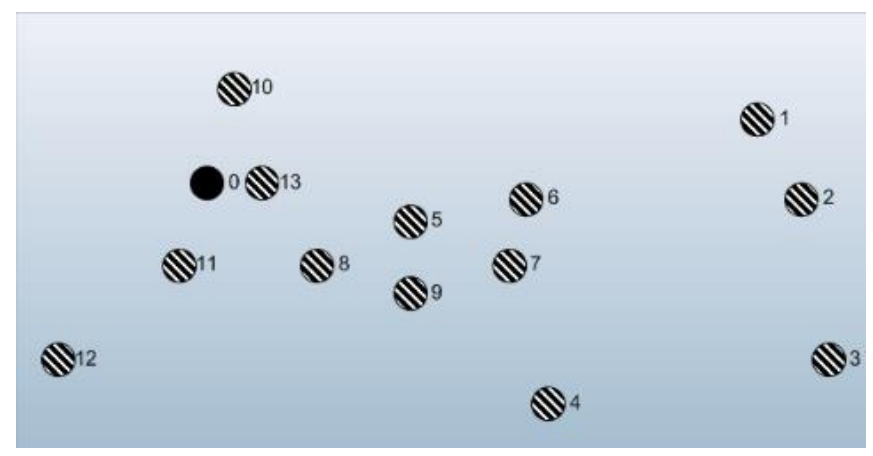

Figure 5. The Topology of the Nodes

\subsection{Results and Evaluation}

Through our previous work, when traditional CTP protocol is applied, some exceptions would appear when the voltage of 2 AA batteries is under $2.3 \mathrm{~V}$, which could be found in Figure 6.

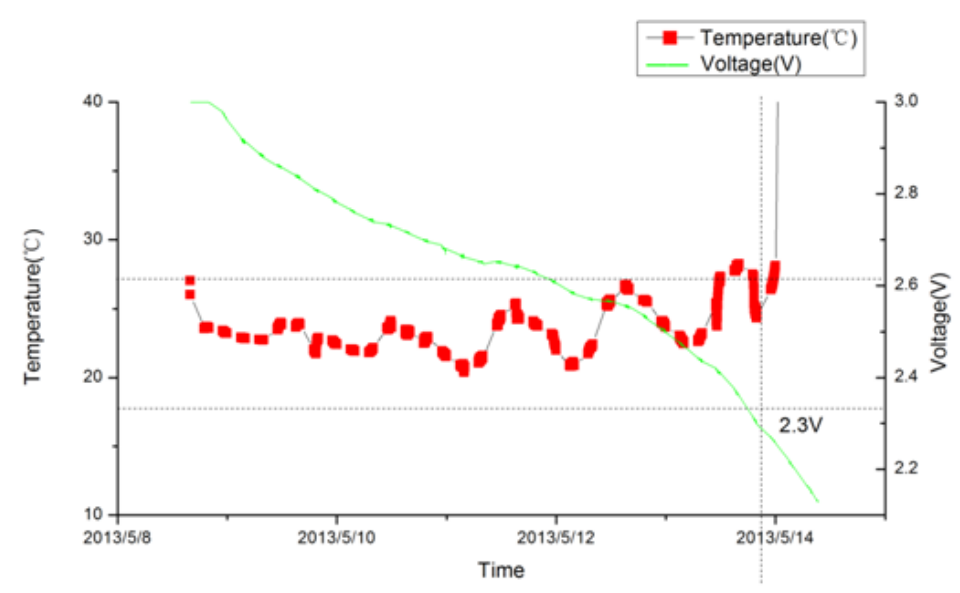

Figure 6. Data Collected Over Time

The red line represents the temperature information collected by the sensor node with a $1 /$ min frequency while the green line reveals the voltage changes with time. It is obvious that the exception would occur when voltage is under $2.3 \mathrm{v}$ in Figure 6 . Herein, we define the terminal voltage to be $2.3 \mathrm{~V}$ and the threshold voltage $2.5 \mathrm{~V}$. When a node's voltage is lower than the threshold value, a special coefficient would be given to reduce the possibility of being selected.

When E-CTP is adopted in the experiment, results of CTP is shown in Figure 7 while ECTP is shown in Figure 8, where all the nodes are given the same initial voltage of $3 \mathrm{v}$, and the same terminal voltage of $2.3 \mathrm{v}$. 


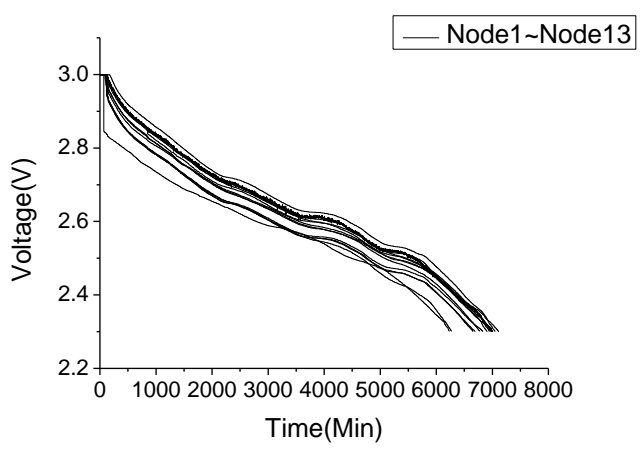

Figure 7. Lifetime in CTP

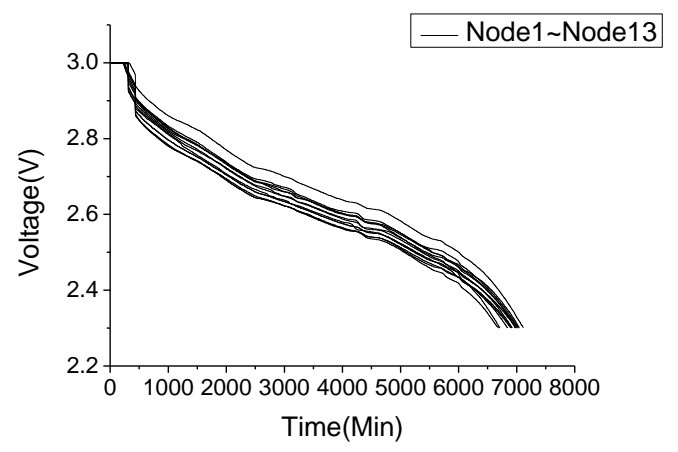

Figure 8. Lifetime in E-CTP

Figure 7 in CTP and Figure 8 in E-CTP show the change of voltage over time. Compared with the voltage in Figure 7 and Figure 8, we can find that the curve in Figure 8 has a more compact form, which means the covariance of voltage in E-CTP is less than that in CTP, as voltage information is taken into consideration in E-CTP. A more specific comparison regarding to lifetime of each node are conducted in Figure 9 and Figure 10 which shows the behavior in the last 1000 minutes. Obviously, when CTP is adopted, some nodes under normal operating voltage emerge after 6000 minutes, while most nodes in E-CTP could last up to 7000 minutes.

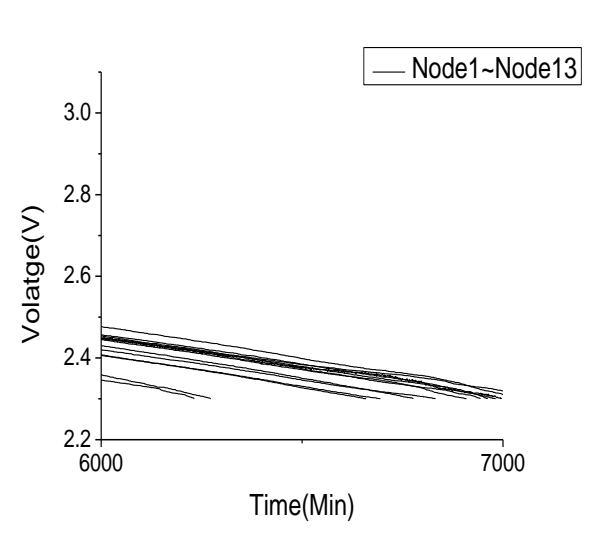

Figure 9. The Detail of Postmortem
Interval in CTP

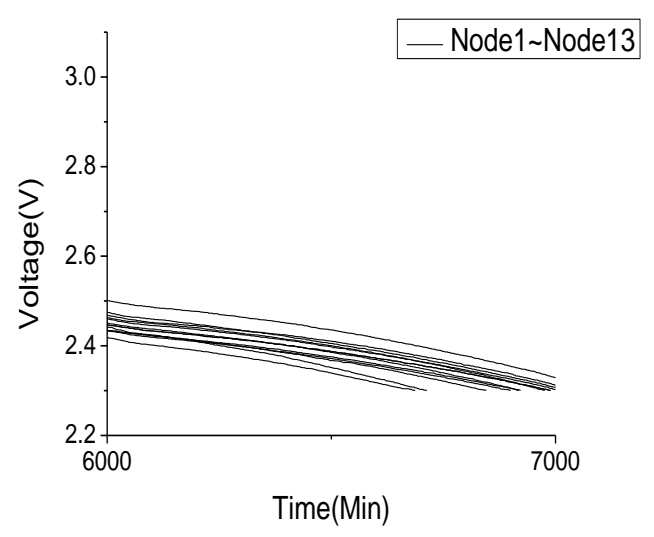

Figure 10. The Detail of Postmortem Interval in E- CTP

As one of our goals is to enhance the lifetime of the network, the comparison histogram of each node is shown in Figure 11, from which we can analyze the specific changes more clearly. 


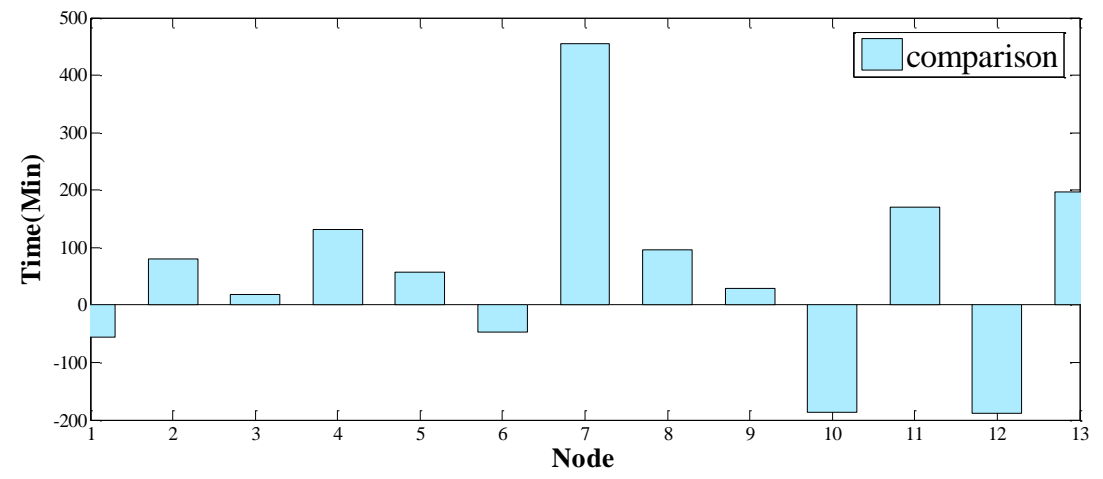

Figure 11. Comparison of each Node

From Figure 11, it could be easily obtained that the work time of most nodes has been extended whilst the range between them has decreased. Meanwhile, a significant change on Node 7 should be noticed. In CTP, compared to other nodes, Node 7 can only survive for 6000 minutes, 100 minutes lower than the average lifetime, which could easily lead to route hole when deployed in large scale. Nevertheless, when E-CTP is adopted, the work time has increased to 500 minutes higher than that of CTP. The voltage comparison of Node7 in different protocols can be found in Figure 12.

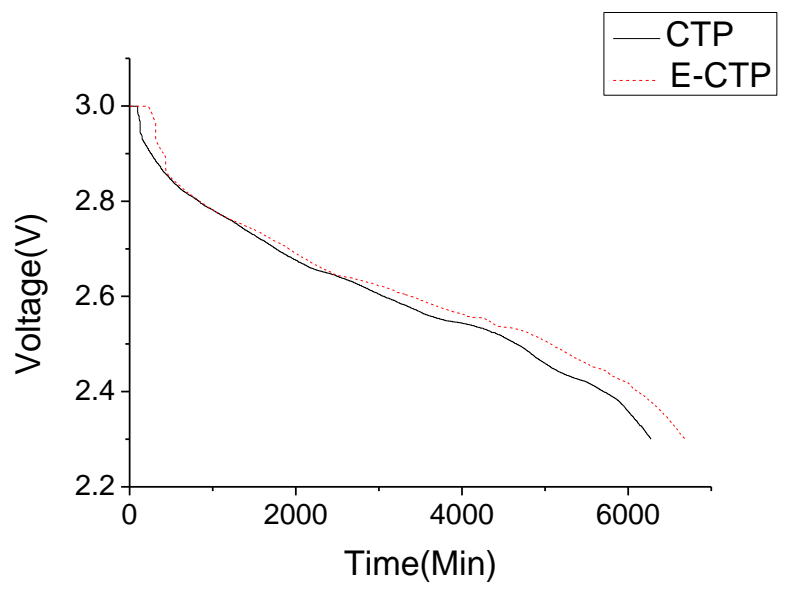

Figure 12. Comparison of Node 7

Once the voltage of each node and the covariance of the whole network are both taken into consideration, E-CTP would have the ability to prevent the excessive energy consumption of any specific node and keep the balance as far as possible. The detail comparison of Node 7 is listed in Table 1.

Table 1

\begin{tabular}{llll}
\hline Index & Minimum & Mean & RMS \\
\hline E-CTP & 6750 & 7008 & 125.57 \\
CTP & 6295 & 6950 & 245.96 \\
\hline
\end{tabular}


From Table 1, we could find that the minimum lifetime in E-CTP has developed from 6295 to 6750 minutes, which could prevent the premature route hole. Also a longer mean lifetime along with a smaller RMS prolongs the lifetime and improves the quality of the network.

\section{Conclusions and Future Work}

TelosB embedded with TinyOS is adopted as the single node in this experiment, and the results shows that compared with the traditional CTP, the E-CTP proposed in this paper could not only ensure reliable communication, but also balance the energy consumption. Moreover, the E-CTP has a prolonged lifetime to avoid the route holes. In fact, the maximum percentage improvement of the single node has reached $7.2 \%$.

Considering that the experiment was conducted in a relative small scale with 14 nodes, the E-CTP would play a greater role in energy balance and high-quality communication when deployed in a larger scale. In the future work, we would also focus on the nodes which could not collect the correct data. When a node is under the terminal voltage, it would be only tasked with forwarding data from other sensors. In this way, it may make the networks more efficient.

\section{Acknowledgements}

This work is supported by the NSFC (61232016, 61173141, 61173142, 61173136, 61103215, 61373132, 61373133, 61100236), National Basic Research Program 973 (2011CB311808), 2011GK2009, GYHY201206033， 201301030， 2013DFG12860, SBC201310569, PAPD fund and Nanjing University of Information Science Research Fund.

\section{References}

[1] I. F. Su Akyildiz, W. Sankarasubramaniam and E. Yogesh Cayirci, "A survey on sensor networks", Communications magazine, IEEE, vol. 40, (2002), pp. 102-114.

[2] J. Yick, Yong, Q. Dong Chen, Y. Ye and X. Wen, "Wireless sensor network survey", Computer networks, vol. 52, (2008), pp. 2292-2330.

[3] L. He Mo, Y. Liu, Y. Zhao, J. Tang, S.-J. Li, X.-Y. Dai and Guojun, "Canopy closure estimates with GreenOrbs: sustainable sensing in the forest", Proceedings of the 7th ACM Conference on Embedded Networked Sensor Systems, (2009), pp. 99-112.

[4] L., J. D. Cayan, D. R. Dettinger and D. Michael, "Meteorology and hydrology in Yosemite National Park: A sensor network application", Information Processing in Sensor Networks, (2003), pp. 518528.

[5] R. Gnawali Fonseca, Clausen, T. Yi and J., "The collection tree protocol", Proceedings of the 7th ACM Conference on Embedded Networked Sensor Systems (SenSys' 09), (2006), pp. 1-14.

[6] R. Gnawali Fonseca, O. Jamieson, K. Kim, S. Levis, P Woo and A., "TEP 123 Collection Tree Protocol (CTP)[OL](2009)", ed.

[7] R. Gnawali Fonseca, O. Jamieson, K. Levis and P., "Tep 119: Collection", Core Working Group, TinyOS Community.

[8] Sharma, D. Liscano, R. Heydari and S. S., "Collector Tree Protocol (CTP) performance in mobile Wireless Sensor Networks", Electrical and Computer Engineering (CCECE), 2011 24th Canadian Conference on, (2011), pp. 001548-001552.

[9] T. Clausen and J. Yi, "Collection Tree Protocol for Lightweight On-demand Ad hoc Distance-vector Routing-Next Generation (LOADng-CT)", (2013).

[10] Y. Elmorsy Gadallah, M. Ibrahim and M. N Ragai, Hani., "ECTP: Enhanced Collection Tree Protocol for practical wireless sensor network applications", Wireless Communications and Mobile Computing Conference (IWCMC), 2013 9th International, (2013), pp. 165-170.

[11] Y. Chen Li, H. He, R. chuan Xie and R. Zou, Shaocong, "ICTP: An improved data collection protocol based OnCTP", Wireless Communications and Signal Processing (WCSP), 2010 International Conference on, (2010), pp. 1-5. 
[12] S. Österlind Duquennoy and A. Fredrik Dunkels, "Lossy links, low power, high throughput", Proceedings of the 9th ACM Conference on Embedded Networked Sensor Systems, (2011), pp. 1225.

[13] Y. He Liu, Y. Li, M. Wang, J. Liu, K. Mo, L. Dong, W. Yang, M. Zheng Xi and J. Zhao, "Does wireless sensor network scale? A measurement study on GreenOrbs", INFOCOM, 2011 Proceedings IEEE, (2011), pp. 873-881.

[14] Q. Dong Chen Yong, Y. Ye and X. Wen, "Lifetime of WSN Research Based on Energy Balance", Applied Mechanics and Materials, vol. 303, (2013), pp. 231-235.

[15] A. Giridhar and P. Kumar, "Maximizing the functional lifetime of sensor networks", Proceedings of the 4th international symposium on Information processing in sensor networks, (2005), pp. 2.

[16] T. Datasheet, "Crossbow Inc", ed.

[17] D. De Couto, S. J. Aguayo, D. Bicket and R. John Morris, "A high-throughput path metric for multihop wireless routing", Wireless Networks, vol. 11, (2005), pp. 419-434.

[18] R. Gnawali Fonseca, O. Jamieson and P. Kyle Levis, "Four-bit wireless link estimation", Proceedings of the Sixth Workshop on Hot Topics in Networks (HotNets VI), (2007).

[19] P. Levis, P. Madden, S. Polastre, J. Szewczyk, R. Whitehouse, K. Woo, A. Gay, D. Hill, J. Welsh and E. Matt Brewer, "TinyOS: An operating system for sensor networks", Ambient intelligence, ed: Springer, (2005), pp. 115-148.

[20] D. Gay, D. Levis, P. Von Behren, R. Welsh, M. Brewer and D. Eric Culler, "The nesC language: A holistic approach to networked embedded systems", Acm Sigplan Notices, (2003), pp. 1-11.

\section{Authors}

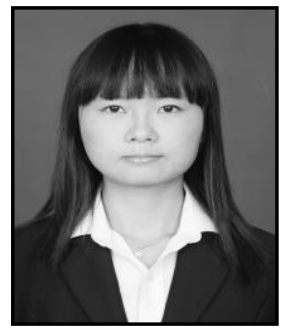

Jingjing Zhang, she received her B.S. degree in Computer Science from Nanjing University of Information Science and Technology, China in 2011. Currently she is studying for her M.S degree in Meteorological Information Security at the same university. Her research interests include wireless networks and network security.

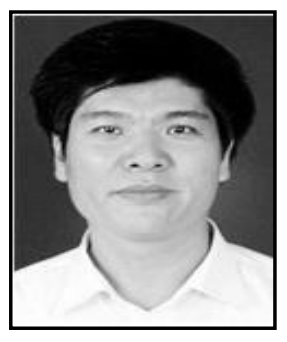

Zhenqi Yang, he is a professor in the School of Computer and Software, Nanjing University of Information Science and Technology, China from 2006. He received the B.S. degree in Applied Mathematics from Qufu Normal University and M.S. degree in Application of mathematical operational research from Chinese academy of science, in 1983and 1988, respectively. From the year 1990 to 2000, he as a visiting scholar of Fudan university. His research interests include information security, Protocol analysis, broadband exchange and cryptography.

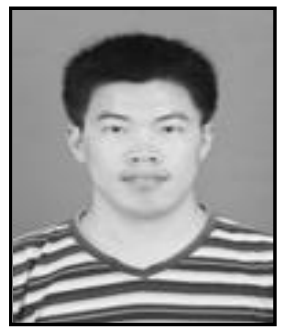

Baowei Wang, he received his B.S. and Ph.D. degrees in Computer Science from Hunan University in 2005 and 2011, respectively. He is currently working as a lecturer in School of Computer and Software, Nanjing University of Information Science and Technology. His research interests include steganography, wireless networks and securing ad hoc networks. 


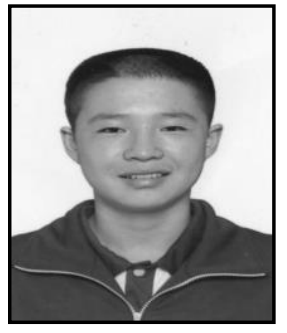

Huiyu Sun, he is a University of Bath undergraduate reading Mathematics. He has spent the last 7 years studying in the United Kingdom and was top of his Maths and Physics classes in secondary school. Prior to the UK, he underwent education in China. He has worked part-time as a computer-modeling assistant in a marketing consultancy in Cambridge, UK.

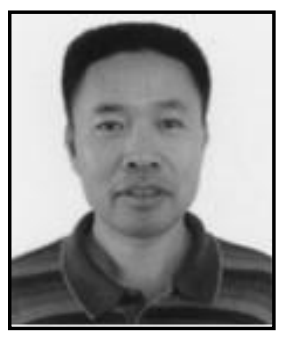

Xingming Sun, he is a professor in the School of Computer and Software, Nanjing University of Information Science and Technology, China from 2011. He received the B.S.degree in Mathematical Science from Hunan Normal University and M.S. degree in Mathematical Science from Dalian University of Technology in 1984 and 1988, respectively. Then, he received the Ph.D. degree in Computer Engineering from Fudan University in 2001. His research interests include information security, network security, cryptography and ubiquitous computing security. 
International Journal of Grid and Distributed Computing Vol.7, No.2 (2014) 